\title{
Localization and Tracking in Sensor Systems
}

\author{
Eric D. Manley, Huzaifa Al Nahas, and Jitender S. Deogun \\ University of Nebraska \\ Department of Computer Science and Engineering \\ Lincoln, Nebraska, United States \\ \{emanley, halnahas, deogun\}@cse.unl.edu
}

\begin{abstract}
In this paper, we survey the current state-of-art of localization and tracking. Our emphasis is on algorithms and systems used in sensor networks. We categorize localization and tracking systems into a hierarchical taxonomy based on characteristics of the objects being tracked, the application environments, and the sensor network technologies. Ideas leading to new directions for future research are briefly discussed.
\end{abstract}

\section{Introduction}

Object tracking and localization has been a topic of interest in many civilian and military applications. Abundant examples for such applications range from assistive technology and robot navigation to search-and-rescue missions and virtual reality systems. Localization is the process of determining the position of an object in space. Tracking seeks to identify the position of an object over time. In some cases, the problem of tracking may be reduced to a series of localization problems. However, localizing a stationary object often differs from localizing a moving object because the moving object exhibits different characteristics. A variety of solutions exist for the localization and tracking problem distinguished by the characteristics of the object, the environment, and the available technology. In this paper, we discuss many of the available systems and algorithms. The topics are categorized into a hierarchy based on the characteristics of the system (see Figure 1).

The first division in the hierarchy breaks up localization and tracking systems into categories based on the type of object. In the layout problem, discussed in Section 2, each object is a stationary device which must work with the other objects to locate itself without any previous location information. In tokenless localization and tracking, discussed in Section 3, the object may be any mobile entity that does not carry any devices used in the localization and tracking algorithm. In token tracking and localization, discussed in Section 4, the object is a mobile device that assists with the localization and tracking. The subcategories are addressed within each section. Future research directions are considered in Section 5.

\section{Layout Problem}

Location-aware applications in sensor networks require each node to know its location in space. This information may be manually distributed, but a solution which bootstraps location information without external assistance is much more attractive. The layout problem, sometimes called the self-configuring localization problem, is the problem of finding coordinates for each node in the network using only estimates of distance between nodes. A solution to this problem may only be correct up to translation, rotation, and reflection; so absolute coordinates may only be realized if three nodes know their absolute locations. While useful in itself, this problem must be solved before many of the tracking algorithms discussed later will work. The following addresses the three main techniques for solving the layout problem: trigonometric configuration, initialization and optimization, and uncertainty-based layout.

\subsection{Trigonometric Configuration}

The first layout technique uses elementary trigonometric properties. The Assumption Based Coordinates (ABC) algorithm [25] first chooses four nodes within communication range of each other. Using trigonometry and the distance measurements between each pair, the four nodes discover their relative locations in an arbitrary coordinate system. New coordinates are assigned incrementally using multilateration from previously localized nodes.

This type of solution has two problems:

1. Inherent error in distance measurements cascade as nodes are incrementally localized. 


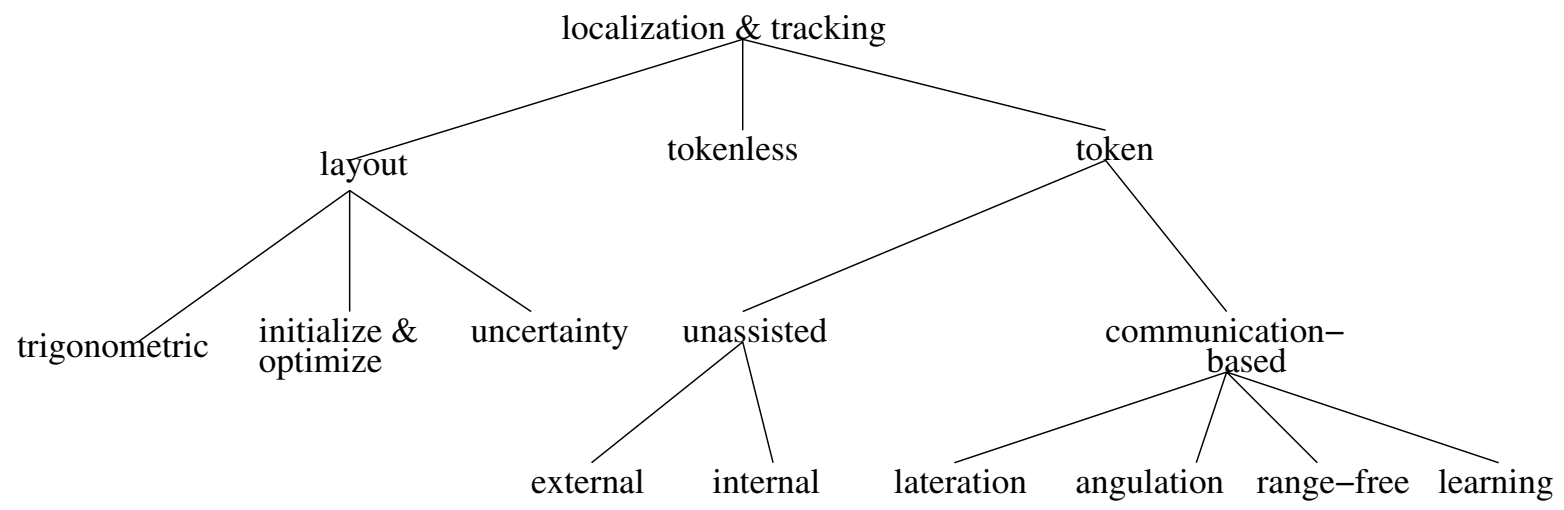

Figure 1. Taxonomy of localization and tracking systems

2. Many layouts cannot be uniquely determined from the distances between nodes. Given a valid layout, large portions of the network may fold over one another admitting another valid layout.

\subsection{Initialization and Optimization}

To overcome these problems, another technique has been developed which runs in two phases $[9,11,14,16,17,22$, 36]. During the first phase, locations are initialized for each device in hopes of achieving the general topology of the network and avoiding problematic fold overs. In the second phase, the predicted locations are iteratively adjusted to minimize global error between the distances admitted in the predicted layout and the measured distances. The resulting allowance of some small error in the predicted layout reduces the impact that error in the measured distances has on the global layout. When processed in a distributed manner, measurement error does not propagate through the network as in the incremental approach.

\subsection{Layout Under Uncertainty}

When sensor nodes are deployed using an airplane or munition launch, accurate data about the positions of anchor nodes may not be available. Rather, the position of these nodes may follow a probability density function. In [19], prior information about node positions and uncertainty of calibration measurements are assumed to be Gaussian, and the layout problem is formulated as a nonlinear minimization problem. If prior information about at least two nodes is known with relatively high-accuracy, then the techniques in [18] may be used to solve the minimization problem. The problem is more challenging when prior information has high uncertainty. An efficient approximation for solving the self-calibration problem in the later case is given in [19].
An approach to the layout problem using Nonparametric Belief Propagation (NBP), a generalization of particle filtering, is given in [14].

\section{Tokenless Localization and Tracking}

In tokenless localization and tracking, the object does not carry any additional devices. All information about location must be inferred from the natural characteristics of the object by devices placed in the environment. The object may be hostile or unaware of the system. When tracking several objects, each object must be identified for tracking information to be useful. This aspect of the problem is itself quite challenging.

The Smart Floor [21] system attempts to identify and track persons based on characteristics of their stride. This system lines the floor with tiles containing pressure sensors that measure the ground reaction force as a person steps on the tile. Machine learning techniques are used to match the pressure data with a person. Once a person is identified, he or she may be tracked based on the location information known a priori about each tile.

The Line in the Sand [2] project seeks to classify and track objects in an outdoor environment using a network of wireless sensing devices. Magnetometers and micro-power impulse radar sensors classify detected objects as soldiers, civilians, or vehicles. The location of each sensing device must be known a priori. Each device routes its location, time, and the classification of a detected object over wireless links to the user. Aggregation of data from several devices allows for tracking. Acoustic, chemical, electric, seismic, optical, and ultrasonic sensors could also be used with the system. 


\section{Token Localization and Tracking}

The localization and tracking of a token (sometimes called a tag) brings an entirely different set of assumptions and requirements to the problem. The token carried by the object is a device such as an RFID tag, a wireless sensor device, a PDA, or a laptop computer that assists in the tracking. Unlike in tokenless tracking, token tracking requires the cooperation of the object. A token may have a unique identifier trivializing the identification or classification problem in Section 3.

In the unassisted tracking problem, the environment is not equipped with devices that aid in tracking the token. With communication-based tracking, devices in the environment may communicate with the token. These variations are discussed next.

\subsection{Unassisted Tracking}

Since no devices in the environment are configured to assist in tracking, the token must collect all needed information on its own. The token may route this information to external destinations, but it relies on no other devices. The token must gather information for inferring location from internal or external sources.

\subsubsection{External Information}

The characteristics of the environment provide some location information. Just as a person knows her location when in a familiar place or a ship navigates by stars at night, a device can estimate location by learning information about its surroundings. Mobile robots often obtain information about location in this manner using sensors [31, 30, 35]. With learning theory, robots can begin to recognize landmarks that assist in navigation [30].

\subsubsection{Internal Information}

Another source of location information exists internally within the device. For instance, consider a ship out at sea during a cloudy night. The stars are unavailable for navigation, but the ship can estimate its velocity and direction. The distance traveled and heading can be computed providing a measurement of displacement. Assuming the initial position of the ship is known, new position estimations can be made continuously. This technique is commonly referred to as dead reckoning.

Dead reckoning techniques have been used extensively for localization and tracking of mobile robots $[32,33]$. Recently, the dead reckoning approach has been applied to the localization and tracking of pedestrians [8]. A three axis accelerometer and magnetic compass are used with offthe-shelf sensor mote technology. The device may operate within a traditional sensor network, but records tracking data while away from the network area. This system works best when the sensors are located close to the foot of the object and learned data about the object's stride is considered. Unfortunately, error propagates through time with dead reckoning systems.

\subsection{Communication-Based Tracking}

With communication-based tracking, devices are deliberately placed in an environment to assist in tracking the token. As with the layout problem in Section 2, some systems measure the distances between devices. This measurement is called ranging and is the basis for lateration systems. Angulation systems are similar, but instead measure angles between devices. Systems that do not use any such measurements are called range-free. Systems that use learning theory by examining the features of communication signals at known locations and then generalizing are called learning systems. Lateration, angulation, range-free, and learning systems are discussed next.

\subsubsection{Lateration Systems}

Using distance measurements between mobile nodes and nodes at known, fixed locations permits the use of multilateration. Robust localization with noisy measurements is often possible. The two main techniques for measuring distance are time-difference-of-arrival, and time-of-flight. Systems using each of these are described next.

Time Difference of Arrival Propagation speeds of different signals vary widely. For example, radio frequency (RF) signals travel much faster than ultrasonic signals. The difference between the arrival of two signals can be used to estimate distance.

The Cricket location-support system [23] uses sensor nodes that have RF radio and ultrasonic receivers. This system relies on two types of nodes: Beacons that are spread all over a building and listeners that are attached to the tokens. When a listener receives an RF signal, it turns on its ultrasonic receiver. The listener uses the time difference between the receipt of the RF and ultrasonic signals to determine the distance to the beacon. Proximity information may be obtained from one beacon, but multilateration may be used for accurate localization when several beacon nodes are in range. There are some limitations to beacon placement in the environment. For instance, if a beacon separates two spaces, it must be placed a fixed distance from the boundary. 
The Cricket system has been tested in both active and passive modes [29]. In passive mode, fixed nodes transmit to tokens whereas in active mode, the tokens transmit to fixed nodes. The active mode provides better accuracy because a beacon signal is received simultaneously by the listeners while in a passive mode a listeners receives beacons signals at different times. Scalability is better in passive mode as more mobile objects can move in the same area and all of them are able to listen to beacon signals. Since a mobile object need not transmit any signals in passive mode, this mode provides better privacy. A hybrid approach of the passive and active mode [29] can provide the advantages of both modes.

Similar systems using time-difference-of-arrival measurements are the DOLPHIN system [10], the SmartLOCUS system [6], and the Ad-Hoc Localization System [26].

Time of Flight The round-trip time-of-flight of a signal can be used for distance estimation.

The PinPoint $3 D-i D[1,34]$ system uses RFID technology for localization and tracking. A battery-operated RFID tag is attached to each mobile object. The tag operates in sleep mode and wakes up periodically to transmit an RF signal. A tag is equipped with motion detector that increases the frequency of transmissions on movement. Using the received signal, readers obtain the tag's distance by measuring the round trip time-of-flight. Readers are organized in cells, with each having a cell controller that is attached to up to 16 antennas.

The Active Bat [12] system uses the time-of-flight of ultrasonic signals to estimate distance. The speed of sound is inferred from the ambient temperature. Ultrasound receivers are distributed in a building by mounting them to the ceiling. The token is referred to as a bat. The bat has an $\mathrm{RF}$ radio and ultrasonic transmitters. A controller sends RF messages simultaneously to both the bats and the ultrasonic receivers. The message sent to the bats informs them to send an ultrasonic pulse. Then the ultrasonic receivers measure the time between receipt of this message and the receipt of the ultrasonic pulse. Distances are then forwarded to a central controller that estimates bat position.

\subsubsection{Angulation Systems}

Angulation systems use an array of either antennas or ultrasonic receivers. An angulation system determines the angle of arrival (AOA) that indicates the direction of a received signal. Not only does this approach help in estimating position, but it also provides orientation capability. Triangulation is used to estimate location from the measured angles.

The Cricket Compass [24] system uses a five passive ultrasonic receivers positioned in a "V" shape to measure AOA. Beacons are placed in the ceiling and transmit their coordinates over the radio. Listeners use the phase difference between the waveforms of receivers to determine differential distance to the beacons. This system is robust to magnetic and time-varying electrical fields that interfere with traditional magnetic compasses.

With the Ad Hoc Positioning System using AOA [20], some nodes are landmarks that know their positions. A node only communicates with its one-hop neighbors. If a node happens to have landmark neighbors, it calculates its orientation and forward that value to its other neighbors. A node that has some neighbors with orientation for a landmark can then estimate its orientation for that landmark. Thus, this system relies on incremental propagation of orientation values over the network.

\subsubsection{Range-Free Localization}

Range-free localization algorithms assume no specific distance measurements among sensor nodes. Instead, proximity to anchor nodes is used. These systems generally provide lower accuracy but with lower cost.

The APIT algorithm [13] can be used when there are some nodes that have ranging capability and high-powered transmitters. The other nodes, called beacon nodes, need not have any ranging capabilities. APIT divides the deployment area of a sensor network into rectangular regions between beacon nodes. By testing whether a node lies within rectangles, node position can be estimated. Localization error of the APIT algorithm decreases when node density increases.

The HS (Hop-Skip) algorithm [5] provides optimal range-free localization in one dimensional space. The GHoST (General Hop Stretcher-Trimmer) algorithm [5] generalizes the HS algorithm to higher dimensions and provides better accuracy than other hop-based localization schemes.

\subsubsection{Learning Systems}

When signal features of fixed nodes are recorded at known locations, the rich theory of machine learning can be used to infer new locations. Even though location information is required, specific distances are not computed, so these algorithms may also fall into the range-free category.

Slobodan N. Simić applies learning theory to several applications in wireless sensor networks including localization and tracking [28]. Simić states the localization problem so that some nodes know their locations and some do not. The nodes which know their location gather training data by communicating with each other. Applying regression techniques, the locations of the other nodes are determined. This basic concept is applied to several tracking systems.

Most learning-based tracking systems collect RF signal strength data at known locations which is used to infer lo- 
cations from measurements at new locations. The RADAR system uses the $k$-Nearest Neighbor algorithm for tracking in the 802.11 framework [3]. Artificial neural networks and Bayesian networks have also been examined in this situation $[4,7]$.

The MoteTrack project attempted to take the ideas of RADAR and implement them on sensor mote technology with greater robustness and decentralization [15]. MoteTrack provides an adaptive method for selecting $k$ in the $k$-Nearest Neighbor algorithm.

\section{Research Directions}

Each of the systems and techniques discussed in this survey could be improved. Increasing accuracy and adapting systems to specific applications warrants future investigation in itself. However, some areas require significant research. These issues are discussed in this section.

\section{$5.1 \quad$ Layout Issues}

Because a good layout of stationary nodes is a prerequisite for many other tracking algorithms, the layout problem is arguably the most important area demanding further attention. The major problem with current layout solutions is that many of them perform poorly for certain network topologies. Better algorithms need to be constructed that work well with more networks. Other issues that need to be addressed include finding the best way to measure global error, finding the limit measurement error imposes on global error, and finding better heuristics for the layout problem in geometric graphs (which is NP-Hard [27]).

\subsection{Expanding Localization to Tracking}

Tracking may be reduced to a series of localization problems. For this reason, much of the research presented here addresses the localization problem without much thought on its generalization to tracking. Because mobile objects exhibit different characteristics than stationary objects, this generalization often results in high tracking error. Combining dead reckoning techniques with trilateration or other localization algorithms provides promise. With dead reckoning information, localization algorithms may adjust parameters adaptively to allow for improved tracking. Location histories may also be exploited and used to predict future movements.

\subsection{Indoor Tokenless Tracking}

At this time, it seems that there exist no scalable systems for identification, classification, and fine-grained localization and tracking of humans in indoor environments. The
Smartfloor [21] study is a step in the right direction but the number of sensors needed, the cost, and the amount of configuration required often prohibit its use. It also may be easily fooled by a changing gait. Measuring features like the thermal signature may be more desirable. Further research must be conducted to better solve this problem. The best solutions would be able to detect events such as an elderly person who has fallen which could be used with an automated alarm system.

\section{Conclusion}

As we have seen, there is a rich set of theoretical and practical research supporting localization and tracking in a variety of sensor systems. Some of the research discussed solves the localization problem for a network of stationary nodes, and some provide accurate tracking given an accurate layout. Some of the systems are intended for use with mobile robots but can be coupled with communicationbased systems to provide better accuracy and services. We hope that our discussion points to gaps between the needs of applications and the available systems and leads to good research questions.

\section{References}

[1] Pinpoint asset management solutions http://www.pinpointco.com, December 2005.

[2] A. Arora, P. Dutta, S. Bapat, V. Kulathumani, H. Zhang, V. Naik, V. Mittal, H. Cao, M. Gouda, Y. Choi, T. Herman, S. Kulkarni, U. A. M. Nesterenko, A. Vora, and M. Miyashita. Line in the sand: A wireless sensor network for target detection, classification, and tracking. Technical Report OSU-CISRC-12/03-TR71, Ohio State University, 2003.

[3] P. Bahl and V. N. Padmanabhan. RADAR: An in-building RF-based user location and tracking system. In INFOCOM (2), pages 775-784, 2000.

[4] R. Battiti, T. L. Nhat, and A. Villani. Location-aware computing: a neural network model for determining location in wireless lans. Technical Report DIT-02-0083, University of Trento, February 2002.

[5] R. Bischoff and R. Wattenhofer. Analyzing connectivitybased multi-hop ad-hoc positioning. In PERCOM '04: Proceedings of the Second IEEE International Conference on Pervasive Computing and Communications (PerCom'04), page 165, Washington, DC, USA, 2004. IEEE Computer Society.

[6] C. Brignone, T. Connors, G. Lyon, and S. Pradhan. Smartlocus: An autonomous, self-assembling sensor network for indoor asset and systems management. Technical Report HPL-2003-41, HP Labs, 2003.

[7] P. Castro, P. Chiu, T. Kremenek, and R. R. Muntz. A probabilistic room location service for wireless networked environments. In UbiComp '01: Proceedings of the 3rd interna- 
tional conference on Ubiquitous Computing, pages 18-34, London, UK, 2001. Springer-Verlag.

[8] L. Fang, P. Antsaklis, L. Montestruque, B. McMickell, M. Lemmon, Y. Sun, H. Fang, I. Koutroulis, M. Haenggi, M. Xie, and X. Xie. Design of a wireless dead reckoning pedestrian navigation system : The navmote experience. IEEE Transactions on Instrumentation and Measurement, 2005.

[9] S. P. Fekete, A. Kroeller, D. Pfisterer, S. Fischer, and C. Buschmann. Neighborhood-based topology recognition in sensor networks. ArXiv Computer Science e-prints, pages 5058-+, May 2004.

[10] Y. Fukuju, M. Minami, H. Morikawa, and T. Aoyama. Dolphin: An autonomous indoor positioning system in ubiquitous computing environment. In WSTFEUS, pages 53-56, 2003.

[11] G. Gotsman and Y. Koren. Distributed graph layout for sensor networks. Journal of Graph Algorithms and Applications, 9(1):1-1, 2005.

[12] A. Harter, A. Hopper, P. Steggles, A. Ward, and P. Webster. The anatomy of a context-aware application. In MobiCom '99: Proceedings of the 5th annual ACM/IEEE international conference on Mobile computing and networking, pages 5968, New York, NY, USA, 1999. ACM Press.

[13] T. He, C. Huang, B. M. Blum, J. A. Stankovic, and T. Abdelzaher. Range-free localization schemes for large scale sensor networks. In MobiCom '03: Proceedings of the 9th annual international conference on Mobile computing and networking, pages 81-95, New York, NY, USA, 2003. ACM Press.

[14] A. T. Ihler, J. W. Fisher III, R. L. Moses, and A. S. Willsky. Nonparametric belief propagation for self-calibration in sensor networks. IEEE Journal of Selected Areas in Communication, 2005.

[15] K. Lorincz and M. Welsh. Motetrack: A robust, decentralized approach to RF-based location tracking. In Proceedings of the International Workshop on Location and ContextAwareness (LoCA 2005), May 2005.

[16] J. Ma, Y. Zhu, Q. Chen, and L. M. Ni. Quality-based anchorfree localization with refinement in sensor networks. In International Conference on Computer Networks and Mobile Computing, 2005.

[17] D. Moore, J. Leonard, D. Rus, and S. Teller. Robust distributed network localization with noisy range measurements. In SenSys '04: Proceedings of the 2nd international conference on Embedded networked sensor systems, pages 50-61, New York, NY, USA, 2004. ACM Press.

[18] R. Moses, D. Krishnamurthy, and R. Patterson. A selflocalization method for wireless sensor networks. EURASIP Journal on Applied Signal Processing, 2002.

[19] R. Moses and R. Patterson. Self-calibration of sensor networks. In Proceedings of SPIE, Unattended Ground Sensor Technologies and Applications IV, volume 4743, April 2002.

[20] D. Niculescu and B. R. Badrinath. Ad hoc positioning system (aps) using aoa. In INFOCOM, 2003.

[21] R. J. Orr and G. D. Abowd. The smart floor: a mechanism for natural user identification and tracking. In $\mathrm{CHI}$ '00: CHI 'OO extended abstracts on Human factors in computing systems, pages 275-276, New York, NY, USA, 2000. ACM Press.
[22] N. B. Priyantha, H. Balakrishnan, E. Demaine, and S. Teller. Anchor-free distributed localization in sensor networks. Technical Report TR-892, MIT LCS, April 2003.

[23] N. B. Priyantha, A. Chakraborty, and H. Balakrishnan. The cricket location-support system. In MobiCom '00: Proceedings of the 6th annual international conference on Mobile computing and networking, pages 32-43, New York, NY, USA, 2000. ACM Press.

[24] N. B. Priyantha, A. K. Miu, H. Balakrishnan, and S. Teller. The cricket compass for context-aware mobile applications. In MobiCom '01: Proceedings of the 7th annual international conference on Mobile computing and networking, pages 1-14, New York, NY, USA, 2001. ACM Press.

[25] C. Savarese, J. Rabaey, and J. Beutel. Locationing in distributed ad-hoc wireless sensor networks. In proceedings of IEEE International Conference on Acoustics, Speech, and Signal Processing (ICASSP), pages 2037-2040, May 2001.

[26] A. Savvides, C. C. Han, and M. B. Srivastava. Dynamic fine-grained localization in ad-hoc wireless sensor networks. In proceedings of the International Conference on Mobile Computing and Networking (MobiCom), July 2001.

[27] J. B. Saxe. Embeddability of weighted graphs in $k$-space is strongly np-hard. In Proceedings of the 17th Allerton Conference on Communications, Control, and Computing, pages 480-489, 1979.

[28] S. N. Simić. A learning-theory approach to sensor networks. Pervasive Computing, 2(4):44-49, October - December 2003.

[29] A. Smith, H. Balakrishnan, M. Goraczko, and N. Priyantha. Tracking moving devices with the cricket location system. In MobiSys '04: Proceedings of the 2nd international conference on Mobile systems, applications, and services, pages 190-202, New York, NY, USA, 2004. ACM Press.

[30] S. Thrun. Bayesian landmark learning for mobile robot localization. Mach. Learn., 33(1):41-76, 1998.

[31] S. Thrun, D. Fox, W. Burgard, and F. Dellaert. Robust monte carlo localization for mobile robots. Artificial Intelligence, 128(1-2):99-141, 2000.

[32] C.-C. Tsai. A localization system of a mobile robot by fusing dead-reckoning and ultrasonic measurements. In Proceedings of the IEEE Instrumentation and Measurement Technology Conference (IMTC/98), pages 144-149, 1998.

[33] H.-J. von der Hardt, D. Wolf, and R. Husson. The dead reckoning localization system of the wheeled mobile robot romane. In Proceedings of the 1996 IEEE ISICEIRSJ International Conference on Multisensor Fusion and Integration for Intelligent Systems, pages 603-610, 1996.

[34] J. Webr and C. Lanzl. Designing a positioning systems for finding things and people indoors. IEEE Spectr., 35(9):7178, 1998.

[35] B. Yamauchi and R. Beer. Spatial learning for navigation in dynamic environments. IEEE Transactions on Systems, Man, and Cybernetics - Part B: Cybernetics, Special Issue on Learning Autonomous Robots, 26(3):496-505, 1996.

[36] A. Youssef, A. Agrawala, and M. Younis. Accurate anchorfree node localization in wireless sensor networks. In 24th IEEE International Performance, Computing, and Communications Conference (IPCCC), pages 465-470, April 2005. 